\title{
On the longitudinal structure function in the dipole model
}

\author{
Marek Niedziela $^{(1),(2)}$, Michal Praszalowicz ${ }^{(1)}$ \\ (1) M. Smoluchowski Institute of Physics, Jagiellonian University, \\ S. Łojasiewicza 11, 30-348 Kraków, Poland. \\ ${ }^{2}$ Present address: Institute for Experimental Physics, University of Hamburg, \\ Luruper Chaussee 149, D-22761 Hamburg, Germany.
}

\begin{abstract}
We compare new HERA data for the longitudinal structure function $F_{\mathrm{L}}$ with the predictions of different variants of the dipole model. In particular we show that the ratio $F_{\mathrm{L}} / F_{2}$ is well described by the dipole models and is rather insensitive to the details of the fit. Fits to $F_{2}$ are performed with the help of geometrical scaling (GS). Using the property of GS we derive the bounds for $F_{\mathrm{L}} / F_{2}$ both for the different versions of the dipole model and in the general case. Finally we briefly discuss how the higher Fock components of the photon wave function may affect these bounds.
\end{abstract}

PACS numbers: $13.85 . \mathrm{Ni}, 12.38 . \mathrm{Lg}$

\section{Introduction}

Recently H1 [1] and ZEUS 22 Collaborations have published new data on the longitudinal structure function $F_{\mathrm{L}}\left(x, Q^{2}\right)$ in deep inelastic ep scattering (DIS). The H1 analysis extends and improves previous data [3], which now cover kinematical range from $Q^{2}=1.5 \mathrm{GeV}^{2} / c^{2}$ and $x=0.279 \times 10^{-4}$ up to $Q^{2}=800 \mathrm{GeV}^{2} / c^{2}$ and $x=0.0322$. ZEUS data has been taken in much smaller region from $Q^{2}=9$ up to $Q^{2}=110 \mathrm{GeV}^{2} / c^{2}$ (see [4] for summary). In both data sets there is strong correlation between $Q^{2}$ and $x$ values; for each $Q^{2}$ structure function $F_{\mathrm{L}}$ (and also $F_{2}$ that has been measured in the same kinematical points) has been measured over a limited $x$ range, with small $x$ 's concentrated around small values of $Q^{2}$, see Fig. 1. Moreover, since $F_{\mathrm{L}}$ is difficult to extract experimentally, even recent improved data has still large errors.

Longitudinal structure function is of particular interest since, in the first approximation of the parton model, it is equal identically zero [5] (CallanGross relation) and therefore it is generated entirely by radiative corrections. 
On the contrary, in the dipole model $F_{\mathrm{L}}$ is nonzero, albeit small. Indeed, Nachtmann and collaborators have shown that in the dipole model there exists a strict bound that [6, 7, 8 ]

$$
F_{\mathrm{L}} \leq g_{\max } \times F_{2}=0.27 \times F_{2} .
$$

This result, hereafter referred to as an EMNS bound, is independent of the dipole-proton cross-section, and - strictly speaking - follows solely from the properties of the photon- $\bar{q} q$ wave function.
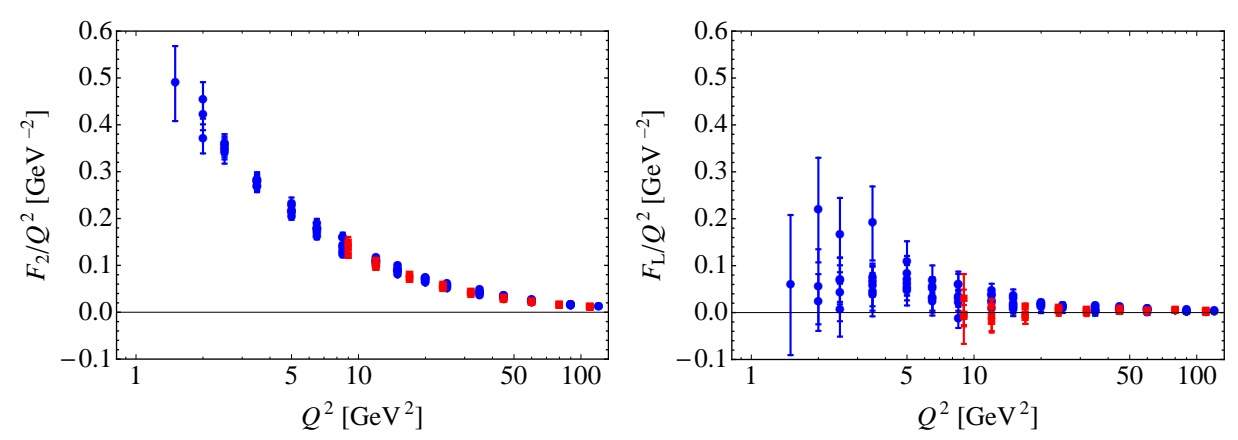

Fig. 1. H1 (blue circles) and ZEUS (red squares) data [1, 2] for $F_{2} / Q^{2}$ (left) and $F_{\mathrm{L}} / Q^{2}$ (right) plotted as functions of $Q^{2}$. Different points for one value of $Q^{2}$ correspond to different Bjorken $x$ 's.

Using previous $\mathrm{H} 1$ data 3 the authors of Ref. 8 , have shown that the bound (1) was almost saturated, which is difficult to realize in realistic dipole models. In this paper we are going to check if this conclusion remains still valid for the new data and what is the value of bound (1) for commonly used dipole-proton cross-sections. Similar analysis for the Golec-BiernatWüsthoff model [9, 10] has been already performed in Ref. [11]. To simplify the analysis we use here the property of geometrical scaling [12] (GS) which is to large precision exhibited by the DIS data up to relatively large Bjorken $x$ 's [13. We find that for realistic dipole-proton cross-sections the bound is indeed lower than (1) with $g \simeq 0.22$ (i.e 18.5\% below the bound of Eq. (1)). In reality these bounds would be lowered if charm quark mass was included.

With present experimental accuracy we do not find any significant tension between $F_{\mathrm{L}} / F_{2}$ data and the dipole model. Should such tension arise when new data appear, higher order corrections to the dipole model might resolve the issue. Therefore we discuss a possibility that corrections to the dipole model coming from higher Fock states in the virtual photon may change (1). Higher Fock components are needed e.g. in the dipole model [9] description of the diffractive data [10]. We show that this is possible only if there exists a bound for higher Fock components that is analogous to (1), 
but with longitudinal contribution to $F_{2}$ that is significantly different than in the case of the $\bar{q} q$ state. Only explicit calculation of the $\bar{q} q g$ contribution to the virtual photon wave function might give here a definite answer. Such calculations have been recently carried out by various authors [14, 15, 16], however these results have not been so far applied to the phenomenological analysis of DIS. Although the calculation of $F_{\mathrm{L}}^{\bar{q} q g} / F_{2}^{\bar{q} q g}$ with the help of Refs. [15, 16] might be probably possible, it is beyond the scope of the present paper.

The paper is organized as follows. In Sect. 2 we rederive the EMNS bound using geometrical scaling. Next, in Sect. 3, we fit two versions of the dipole model to the present data set for $F_{2}$. To this end we also use the property of geometrical scaling. We then compare these fits with the data for $F_{\mathrm{L}}$ and discuss fit uncertainties. In Sect. 4 we calculate ratio $F_{\mathrm{L}} / F_{2}$ for the afore mentioned fits and compare it with the data and with the EMNS bound. We do not find large tension between the data and model predictions. An influence of higher Fock states on the EMNS bound is discussed in Sect. 5. We conclude in Sect. 6.

\section{Geometrical scaling and the EMNS bound}

For three massless flavors DIS structure functions read [17]:

$$
\begin{aligned}
& F_{2}\left(x, Q^{2}\right)=\frac{Q^{2}}{4 \pi^{3}} \int d r^{2}\left\{\left|\psi_{\mathrm{T}}\left(r, Q^{2}\right)\right|^{2}+\left|\psi_{\mathrm{L}}\left(r, Q^{2}\right)\right|^{2}\right\} \sigma_{\mathrm{dp}}\left(r^{2}\right), \\
& F_{\mathrm{L}}\left(x, Q^{2}\right)=\frac{Q^{2}}{4 \pi^{3}} \int d r^{2}\left|\psi_{\mathrm{L}}\left(r, Q^{2}\right)\right|^{2} \sigma_{\mathrm{dp}}\left(r^{2}\right)
\end{aligned}
$$

where photon wave functions take the following form

$$
\begin{aligned}
\left|\psi_{\mathrm{T}}\left(r, Q^{2}\right)\right|^{2} & =\int_{0}^{1} d z\left[z^{2}+(1-z)^{2}\right] \bar{Q}^{2} K_{1}^{2}(\bar{Q} r), \\
\left|\psi_{\mathrm{L}}\left(r, Q^{2}\right)\right|^{2} & =4 \int_{0}^{1} d z z(1-z) \bar{Q}^{2} K_{0}^{2}(\bar{Q} r) .
\end{aligned}
$$

Here $K_{i}$ are modified Bessel functions and

$$
\bar{Q}^{2}=z(1-z) Q^{2}
$$

It is convenient to define functions $\Phi_{\mathrm{T}, \mathrm{L}}$

$$
\Phi_{\mathrm{T}, \mathrm{L}}(u=r Q)=r^{2}\left|\psi_{\mathrm{T}, \mathrm{L}}\left(r, Q^{2}\right)\right|^{2}
$$


that depend only on the combined variable $u=Q r$. Therefore

$$
\begin{aligned}
& F_{2}\left(x, Q^{2}\right)=\frac{Q^{2}}{2 \pi^{3}} \int d u\left\{\Phi_{\mathrm{T}}(u)+\Phi_{\mathrm{L}}(u)\right\} \frac{\sigma_{\mathrm{dp}}(u / Q)}{u}, \\
& F_{\mathrm{L}}\left(x, Q^{2}\right)=\frac{Q^{2}}{2 \pi^{3}} \int d u \Phi_{\mathrm{L}}(u) \frac{\sigma_{\mathrm{dp}}(u / Q)}{u} .
\end{aligned}
$$

This parametrization is very convenient for the following reasons. First of all wave functions $\Phi_{\mathrm{T}, \mathrm{L}}(u)$ are universal and do not depend on external kinematical variables. Secondly, unlike functions $\psi_{\mathrm{T}, \mathrm{L}}\left(r, Q^{2}\right)$, they are everywhere regular in $u$. And finally, cross-section $\sigma_{\mathrm{dP}}(u / Q) / u$ is a localized function of variable $u$ that vanishes both for $u \rightarrow 0$ and $u \rightarrow \infty$.

If - as it is in the case of the GBW model - the dipole-proton crosssection exhibits geometrical scaling, i.e. $\sigma_{\mathrm{dP}}(r)=\sigma_{\mathrm{dP}}\left(r Q_{\mathrm{s}}(x)\right)$ then the integral

$$
\int d u \Phi_{\mathrm{T}, \mathrm{L}}(u) \frac{\sigma_{\mathrm{dp}}(u / Q)}{u}=\int d u \Phi_{\mathrm{T}, \mathrm{L}}(u) \frac{\sigma_{\mathrm{dp}}\left(u Q_{\mathrm{s}} / Q\right)}{u}=\operatorname{function}(\tau)
$$

depends only on a scaling variable

$$
\tau=\left(Q / Q_{\mathrm{s}}\right)^{2} .
$$

Here $Q_{\mathrm{s}}^{2}$ is a saturation scale

$$
Q_{\mathrm{s}}^{2}=Q_{0}^{2}\left(\frac{x}{x_{0}}\right)^{-\lambda}
$$

Now we can rederive the EMNS bound by considering the ratio

$$
\frac{F_{\mathrm{L}}(\tau)}{F_{2}(\tau)}=\frac{\int d u \Phi_{\mathrm{L}}(u) \sigma_{\mathrm{dp}}(u / Q) / u}{\int d u\left\{\Phi_{\mathrm{T}}(u)+\Phi_{\mathrm{L}}(u)\right\} \sigma_{\mathrm{dp}}(u / Q) / u}
$$

and observing that function

$$
g(u)=\frac{\Phi_{\mathrm{L}}(u)}{\Phi_{\mathrm{T}}(u)+\Phi_{\mathrm{L}}(u)},
$$

which is plotted in Fig. 2, has a maximum $g_{\max }=0.2714$ for $u=2.591$. Therefore it follows that

$$
\frac{F_{\mathrm{L}}\left(x, Q^{2}\right)}{F_{2}\left(x, Q^{2}\right)} \leq g_{\max }=0.27 .
$$




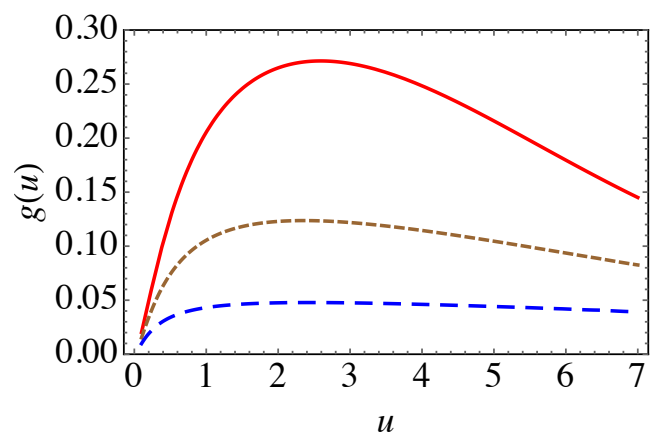

Fig. 2. Solid (red) line: function $g(u)$ defined in Eq. (10). Short dashed (brown) line: function $g(u)$ in the case of massive quarks for $\delta_{\mathrm{f}}=m_{\mathrm{f}} / Q=0.5$ and long dased (blue) line: for $\delta_{\mathrm{f}}=m_{\mathrm{f}} / Q=1.06$, which corresponds to the charm quark mass $m_{\mathrm{c}}=1.3 \mathrm{GeV} / c$ and $Q^{2}=1.5 \mathrm{GeV}^{2} / c^{2}$.

The bound (11) has been derived for the case of massless quarks. While this is certainly a good approximation for three light flavors, given the fact that the lowest photon virtuality in the data set we use is $Q^{2}=1.5 \mathrm{GeV}^{2} / c^{2}$, the inclusion of charm mass effects is going to change (11). For a given flavor function $g$ defined in Eq. (10) is no longer a function of scaling variable $u$ only but in addition depends on the ratio $\delta_{\mathrm{f}}^{2}=m_{\mathrm{f}}^{2} / Q^{2}$. For large $Q^{2}$ (i.e. small $\left.\delta_{\mathrm{f}}\right) g\left(u, \delta_{\mathrm{f}}\right) \rightarrow g(u)$. Moreover we have found numerically that everywhere in $u$ we have

$$
g\left(u, \delta_{\mathrm{f}}\right) \leq g(u)
$$

and the maxima $g_{\max }^{(\mathrm{f})}$ of $g\left(u, \delta_{\mathrm{f}}\right)$ are decreasing with increasing $\delta_{\mathrm{f}}$, as illustrated in Fig. 2. This is consistent with the observation of Ref. [6] that $g_{\max }^{(\mathrm{f})}$ is a monotonically growing function of $Q^{2}$. Therfore

$$
0 \leq g_{\max }^{(\mathrm{f})}\left(Q^{2}\right) \leq g_{\max } .
$$

This allows us to estimate the effect of the charm quark on the ratio:

$$
\begin{aligned}
\frac{F_{\mathrm{L}}^{(\text {light }+\mathrm{c})}}{F_{2}^{(\text {light+c })}} & =\frac{F_{\mathrm{L}}+F_{\mathrm{L}}^{(\mathrm{c})}}{F_{2}+F_{2}^{(\mathrm{c})}}=\frac{F_{\mathrm{L}} / F_{2}+F_{\mathrm{L}}^{(\mathrm{c})} / F_{2}^{(\mathrm{c})} F_{2}^{(\mathrm{c})} / F_{2}}{1+F_{2}^{(\mathrm{c})} / F_{2}} \\
& \leq g_{\max } \frac{1+g_{\max }^{(\mathrm{c})} / g_{\max } F_{2}^{(\mathrm{c})} / F_{2}}{1+F_{2}^{(\mathrm{c})} / F_{2}} \leq g_{\max }
\end{aligned}
$$

where the last inequality follows from $(13)$. Note that $F_{2, \mathrm{~L}}$ without any superscript refers to the structure function with light flavors only and that dependence on $Q^{2}$ has been suppressed. We see therefore, in agreement with 


\begin{tabular}{lcccc}
$x_{\max }$ & $\sigma_{0}\left[1 / \mathrm{GeV}^{2}\right]$ & $\lambda$ & $x_{0}$ & $\chi^{2} /$ dof \\
\hline none & 23.68 & 0.389 & 0.010497 & 1.18 \\
0.01 & 27.11 & 0.353 & 0.007786 & 0.87 \\
0.005 & 29.33 & 0.333 & 0.006435 & 0.79 \\
0.0005 & 38.37 & 0.253 & 0.003090 & 0.70 \\
\hline
\end{tabular}

Table 1. Parameters of the GBW model fitted to $F_{2} \mathrm{H} 1$ data up to $x_{\max }$.

Ref. [8], that inclusion of charm lowers the bound on $F_{\mathrm{L}}^{(\text {light+c) }} / F_{2}^{(\text {light+c) }}$ in proportion that depends on $F_{2}^{(\mathrm{c})} / F_{2}$. For $m_{\mathrm{c}}=1.3 \mathrm{GeV} / c$ and for the lowest $Q^{2}$ in the present data set we get numerically $g_{\max }^{(\mathrm{c})} \approx 0.05$ (see Fig. (2) ), which gives $g_{\max }^{(\mathrm{c})} / g_{\max } \approx 0.19$. We do not know what is the fraction of charm in the present data sample, however taking a typical value of $F_{2}^{(\mathrm{c})} / F_{2} \sim 25 \%$, we get that $g_{\max }^{(\text {light }+ \text { c) }} \sim 0.23$. This means that bound 11 is lowered for the lowest $Q^{2}$ bin by approximately $18 \%$ and is approaching (11) for higher $Q^{2}$. In the following we will ignore charm contribution and stick to the bound (11) coming back to this point in Sect. 4 .

\section{Dipole models and geometrical scaling}

In order to check how far the bound (11) overestimates actual predictions of the dipole model with realistic dipole-proton cross-section, we are going to compute ratio (11) for a given $\sigma_{\mathrm{dp}}$ in terms of scaling variable $\tau$. We will see that for commonly used parametrizations of $\sigma_{\mathrm{dp}}$, ratio $F_{\mathrm{L}} / F_{2}$ is a slowly varying function of $\tau$ with a maximum equal approximately $0.216-$ 0.224 , which only slightly depends on the parametrization actually used. To this end we have decided to perform our own fits to the $F_{2}$ data over the restricted kinematical range where the longitudinal structure function $F_{\mathrm{L}}$ has been measured by H1. The reason for this is threefold. Firstly, new data is of much better quality than the previous data to which the dipole model parameters have been fitted. Secondly, we do not aim at a global fit, but rather at a fit which covers only the points where $F_{\mathrm{L}}$ has been measured as well. Therefore fit parameters - as we shall see in the following - will be different from the ones obtained in the global fits. And finally, we have decided to fit the data with the help of geometrical scaling - a procedure not used so far in the fits to the DIS data.

Fitting dipole models to the data becomes very easy when $F_{2}$ depends only upon single scaling variable $\tau$. This happens because points corresponding to one particular value of $Q^{2}$ but different $x$ 's (see "stacks" in Fig. 1) correspond to different values of $\tau$ and are therefore shifted horizontally - if plotted in terms of $\tau$ - by values that are different for different 
$x$ 's. As a consequence dipole model predictions fall on a universal curve (up to an overall normalization $\sigma_{0}$ ), and data fitting consists in changing $\sigma_{0}$ and the parameters defining scaling variable $\tau$, i.e. $x_{0}$ and $\lambda$. By varying these three parameters one forces experimental points to fall on theoretical prediction, rather than by changing theoretical predictions one is trying to reproduce experimental points. Therefore this method is very efficient, as it does not require time consuming recalculations of the theoretical curve.

Even in the case of dipole-proton cross-sections that violate GS by explicit (albeit weak) dependence on $x$, like in the model of Iancu, Itakura and Munier [18, it is still possible to make a GS-like fit by keeping $x$ at some fixed average value $x_{\text {ave }}$ and then study the uncertainty of theoretical predictions by changing $x$ over the range that is covered by experimental data. We shall come back to this point later.

Let us first consider the simplest version of the dipole model, namely the GBW parametrization [9], which - up to an overall normalization $\sigma_{0}-$ takes the following form in terms of scaling variable $\tau$

$$
\frac{\sigma_{\mathrm{dp}}^{\mathrm{GBW}}(u, \tau)}{\sigma_{0}}=1-\exp \left(-\frac{u^{2}}{4 \tau}\right) .
$$

Pluging (15) into Eqs.(5) gives unnormalized theoretical prediction for the structure functions divided by $Q^{2}$, which will be denoted in the following by small characters $f_{2, L}(\tau)$. Experimental data are tabulated in a set of discrete points $\left\{Q_{i}^{2}, x_{i}\right\}$, and we fit three free parameters of the model, $\sigma_{0}, x_{0}$ and $\lambda$, by transforming experimental entries in the following way:

$$
F_{2}\left(x_{i}, Q_{i}^{2}\right) \rightarrow \frac{1}{Q^{2} \sigma_{0}} F_{2}\left(x_{i}, Q_{i}^{2}\right)=f_{2}\left(\tau_{i}=\frac{Q^{2}}{Q_{\mathrm{s}}^{2}\left(x_{i}\right)}\right)
$$

and demanding that they are equal to the theoretical prediction at the pertinent value of scaling variable $\tau_{i}$ with $Q_{\mathrm{s}}$ given by Eq. (8). The results are shown in Table1. Since GS is supposed to work the best for small values of Bjorken $x$ 's we have performed a number of fits restricting the allowed $x$ region up to a maximal value denoted as $x_{\max }$. We see that even without any cut on the maximal value of $x$, i.e. for $x$ as large as 0.0322 (the highest $x$ in the analyzed data set) $\chi^{2}$ of the fit is quite reasonable. By restricting analyzed data to the smaller and smaller range of Bjorken $x$ 's $\chi^{2}$ is getting smaller, but also model parameters vary substantially. Parameter $\sigma_{0}$ is much smaller than in the original fit of Ref. 9] $\sigma_{0}^{\mathrm{GBW}}=23 \mathrm{mb}=59 \mathrm{GeV}^{-2}$. Exponent $\lambda$ approaches the value of Ref. 9 $\lambda^{\mathrm{GBW}}=0.288$ only for small $x_{\max }$ (note that maximal $x$ in Ref. [9] was equal to 0.01 , whereas the lowest $x=6 \times 10^{-6}$ was much below the minimal $x$ of present analysis), otherwise being consistent with model-independent analysis of Ref. [13]. The results 
of the fits, together with the original parametrization of Ref. [9] are plotted in Fig. 3. One can see rather good agreement of fits from Table 1 with the data, and - also quite importantly - good quality of GS of the data.
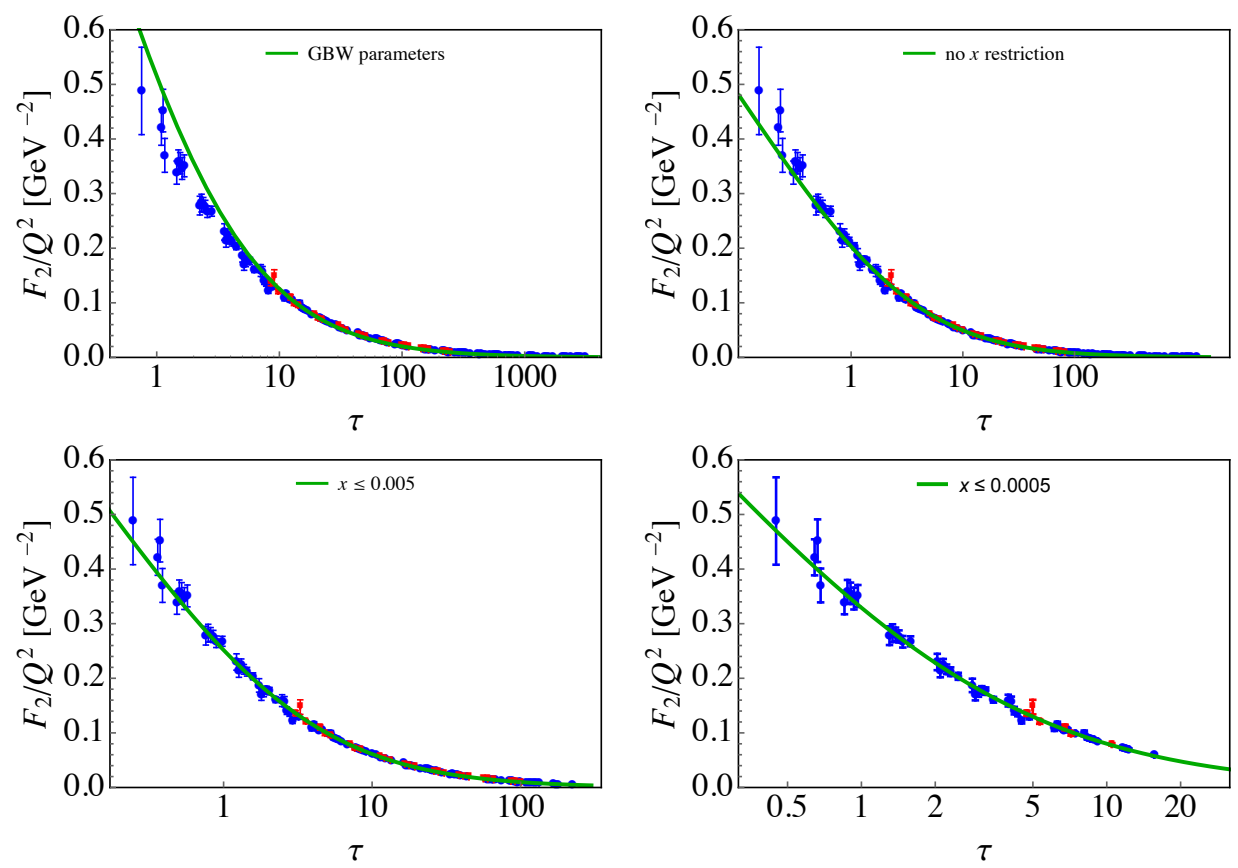

Fig. 3. H1 (blue circles) and ZEUS (red squares) data 1, 2] for $F_{2} / Q^{2}$ plotted as a function of scaling variable $\tau$ for different values of fit parameters corresponding to the GBW model given in Table 1. Fit parameters in the first panel correspond to the original fit of Ref. 9] with no charm data included.

Finally in Fig. 4 we plot data for $F_{\mathrm{L}} / Q^{2}$ as a function of $\tau$ together with theoretical parametrization of Ref. [9] and the present fits corresponding to Tab. 1. We can see that due to still large experimental errors of $F_{\mathrm{L}}$ all parametrizations, although different, describe well the data.

As a second example let us consider a dipole model by Iancu, Itakura and Munier (IIM) [18, where the dipole-proton cross-section is defined in terms of two functions

$$
\begin{aligned}
A_{1}(u, \tau, x) & =A_{0}\left(\frac{u^{2}}{4 \tau}\right)^{\gamma+\frac{\ln \left(4 \tau / u^{2}\right)}{2 \kappa \lambda \ln (1 / x)}}, \\
A_{2}(u, \tau) & =1-\exp \left(-a \ln ^{2}(b u / \sqrt{\tau})\right)
\end{aligned}
$$



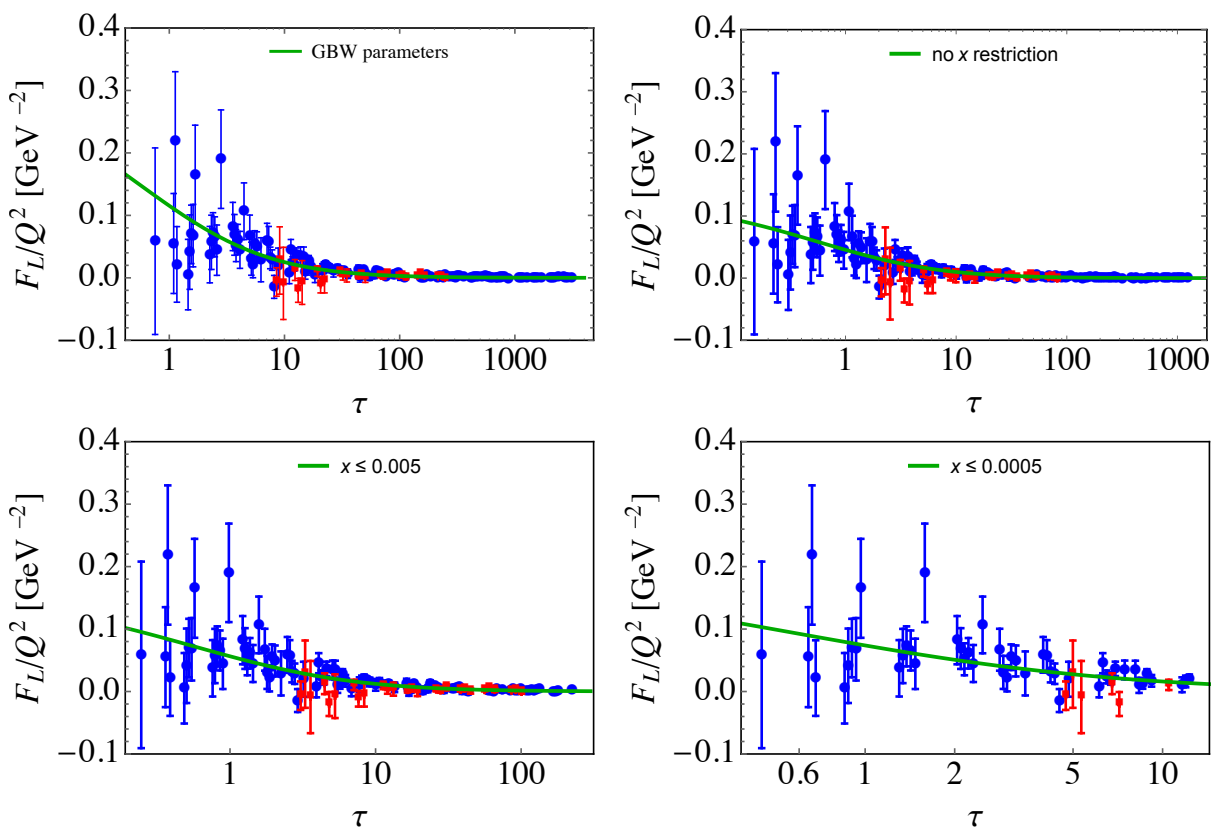

Fig. 4. H1 (blue circles) and ZEUS (red squares) data [1, 2] for $F_{\mathrm{L}} / Q^{2}$ plotted as a function of scaling variable $\tau$. Solid curves correspond to the GBW model fits of Table 1 .

and

$$
\frac{\sigma_{\mathrm{dp}}^{\mathrm{IIM}}(u, \tau, x)}{\sigma_{0}}=\left\{\begin{array}{ccc}
A_{1}(u, \tau, x) & \text { for } & u^{2}<4 \tau \\
A_{2}(u, \tau) & \text { for } \quad 4 \tau \leq u^{2}
\end{array} .\right.
$$

Here $\gamma=0.63$ is related to the anomalous dimension of the forward scattering amplitude in the BFKL formalism, while $\kappa=9.9$ corresponds to the diffusion coefficient. Parameters $a$ and $b$ are determined uniquely by gluing $A_{1}$ and $A_{2}$ and their derivatives at $u^{2}=4 \tau$. Parameter $A_{0}$ is in principle free, however, as it was shown in Ref. [18] the best $\chi^{2}$ was obtained for $A_{0}=0.7$ and for the purpose of the present work we will keep it fixed at this value. Therefore the only free parameters are, as in the case of the GBW model, an overall normalization $\sigma_{0}$ and two parameters of the saturation scale: $\lambda$ and $x_{0}$.

However, there are two important differences between IIM and GBW parametrizations. First of all, for small value of $u$ amplitude $A_{1}$ exhibits explicit violation of GS, since it depends both on $\tau$ and $x$. For the purpose of the present work we will keep $x$ entering $A_{1}$ fixed at the average value $x_{\text {ave }}$ calculated for the interval where the fit is performed. The accuracy of 


\begin{tabular}{llcccc}
$x_{\max }$ & $x_{\text {ave }}$ & $\sigma_{0}\left[1 / \mathrm{GeV}^{2}\right]$ & $\lambda$ & $x_{0}$ & $\chi^{2} /$ dof \\
\hline none & 0.00359 & 20.22 & 0.597 & 0.002553 & 1.76 \\
0.01 & 0.00182 & 21.50 & 0.583 & 0.002140 & 1.57 \\
0.005 & 0.00121 & 25.56 & 0.531 & 0.001392 & 1.31 \\
0.0005 & 0.00022 & 34.30 & 0.389 & 0.000645 & 0.75 \\
\hline
\end{tabular}

Table 2. Parameters of the IIM [18] model fitted to $F_{2}$ H1 data up to $x_{\max }$.
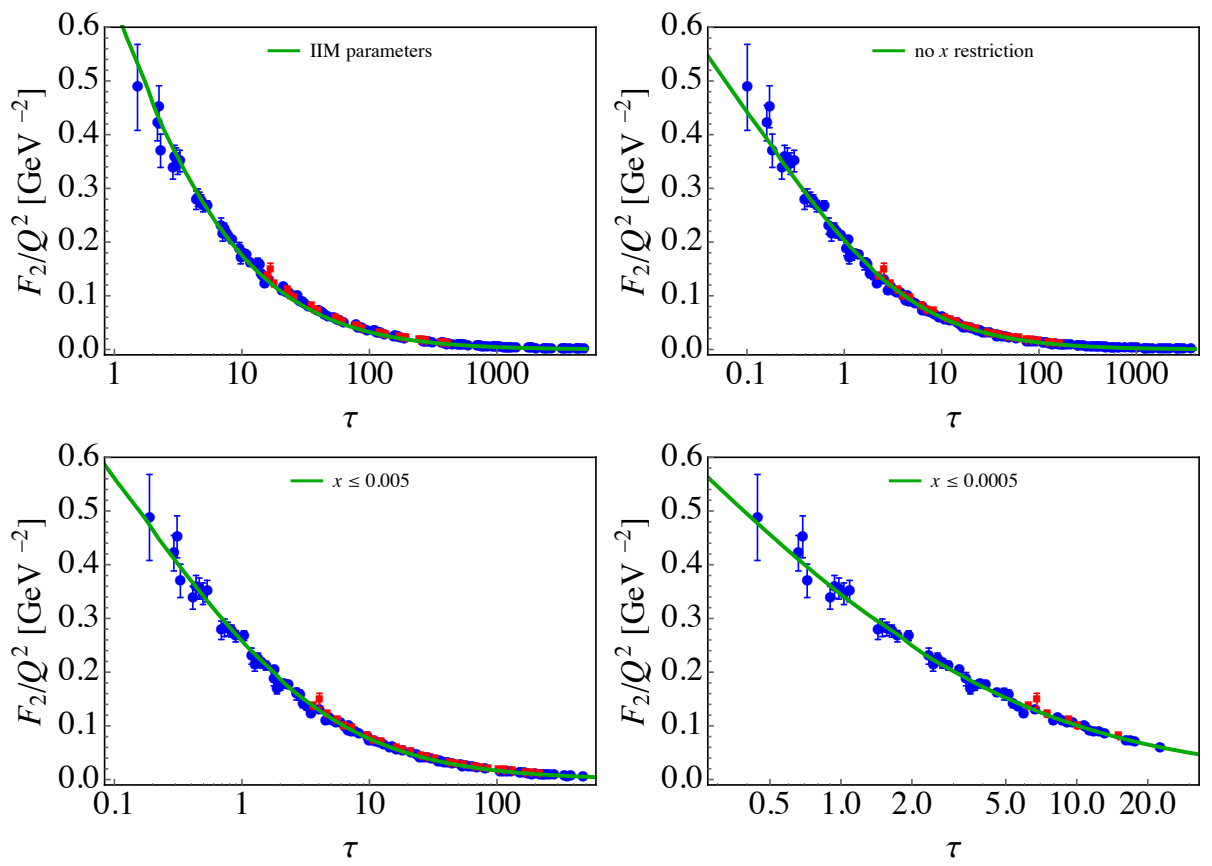

Fig. 5. H1 (blue circles) and ZEUS (red squares) data [1, 2] for $F_{2} / Q^{2}$ plotted as a function of scaling variable $\tau$ for different values of IIM model fit parameters given in Table 2

this procedure is then checked by putting in 180 equal to the maximal and minimal value of $x$ in a given interval. It will turn out that the structure functions are sensitive to this variation of $x$ at the level of a few percent, however the ratio $F_{\mathrm{L}} / F_{2}$ is almost independent. Next difference appears due to the dependence of $A_{1}$ on $\lambda$. To solve this issue we have adopted an iterative procedure, consisting in fixing $\lambda$ at some initial value, then performing the fit and plugging in the fitted value of $\lambda$ to $A_{1}$ and performing the fit again. Usually after four, five steps satisfactory convergence has been achieved. The results are given in Table 2 .

By inspecting Table 2 we see that the quality of fits is worse than in the 


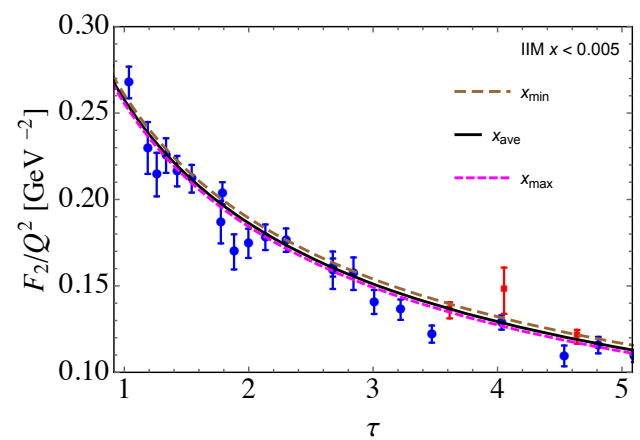

Fig. 6. $F_{2}(\tau) / Q^{2}$ for the parameters from the third row of Table 2 with $x$ in $A_{1}$ from Eq. (17) replaced by $x_{\min }$ (upper curve), $x_{\text {ave }}$ (solid middle curve) and $x_{\max }$ (lower curve).

case of the GBW model. This is in contrast with the original fits of Ref. [18] which, however, were performed over the data set covering much lower $x$ 's than in our present analysis. One should also note that the errors of the old data sets are bigger than the ones of the present data. This is also the reason why fit parameters are different than in our case. For illustration purposes we have plotted in Fig. $5 F_{2}(\tau) / Q^{2}$ for the original IIM parameters and for three choices of $x_{\max }$ from Table 2, Magnifying first plot in Fig. 5 one could see that for $\tau>10$ the original IIM curve missed the experimental points, which have rather small errors.

In order to check sensitivity of the IIM fits to the fact that $x$ dependendent piece of $A_{1}$ amplitude (17) has been replaced by a constant value $x_{\text {ave }}$, we plot in Fig. $6 / F_{2}(\tau) / Q^{2}$ for the parameters from the third row of Table 2 with $x$ in $A_{1}$ replaced by $x_{\min }$ (upper curve) and $x_{\max }$ (lower curve). For better resolution the plot is restricted to $\tau<5$. We can see that theoretical uncertainty introduced by this procedure is in fact much smaller than the experimental errors.

Finally in Fig. 7 we plot $F_{\mathrm{L}} / Q^{2}$ as a function of $\tau$ for three sets of parameters from Table 2 and for the original set of parameters from Ref. [18]. One can see that all curves describe the data reasonably well due to the large error bars of $F_{\mathrm{L}}$.

\section{EMNS bound for dipole models}

Now we are able to compare the EMNS bound with $F_{\mathrm{L}} / F_{2}$ ratio calculated in the dipole model for realistic dipole-proton cross-sections $\sigma_{\mathrm{dp}}$ discussed in the previous Section. One should note that for the GBW dipole model $F_{\mathrm{L}} / F_{2}$ does not depend on the values of $x_{0}, \lambda$ and $\sigma_{0}$. It is not the 

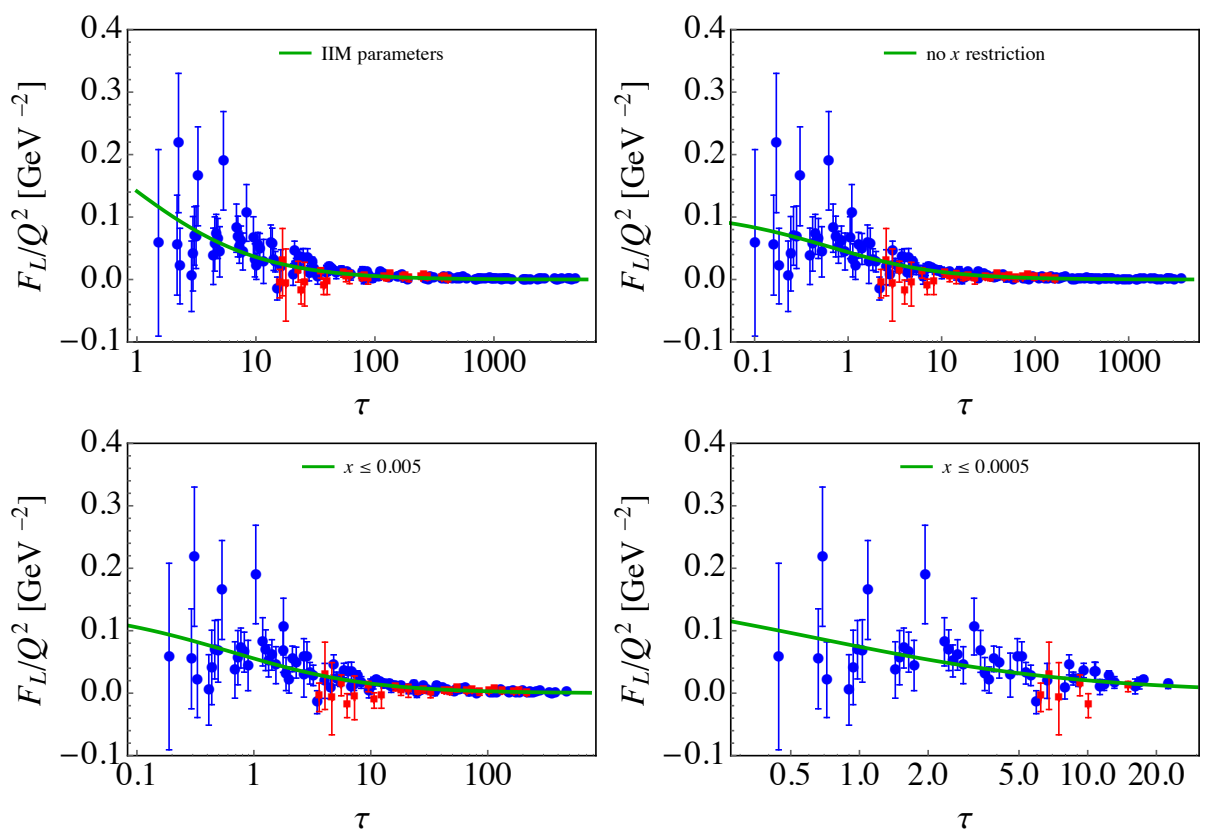

Fig. 7. H1 (blue circles) and ZEUS (red squares) data [1, 2] for $F_{\mathrm{L}} / Q^{2}$ plotted as a function of scaling variable $\tau$. Solid curves correspond to the IIM model fits of Table 2

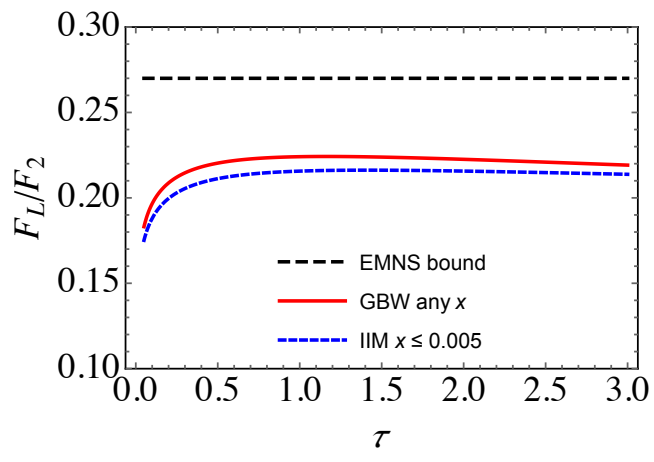

Fig. 8. Ratios $F_{\mathrm{L}} / F_{2}$ as functions of $\tau$ for the GBW dipole model and for the IIM model with parameters corresponding to the fit with $x<0.005$. The scale of the plot has been enlarged in order to make small differences between the two curves visible.

case for the IIM dipole model, but we have checked explicitly that for all parametrizations of Table 2 the differences in $F_{\mathrm{L}} / F_{2}$ are negligible. Therefore in Fig. 8 we plot ratios $F_{\mathrm{L}} / F_{2}$ as functions of $\tau$ for the GBW dipole 


\begin{tabular}{lcc} 
model & $\tau_{\max }$ & $\left(F_{\mathrm{L}} / F_{2}\right)_{\max }$ \\
\hline GBW any & 1.165 & 0.224 \\
IIM all $x$ & 1.417 & 0.217 \\
IIM $x<0.01$ & 1.411 & 0.216 \\
IIM $x<0.005$ & 1.413 & 0.216 \\
IIM $x<0.0005$ & 1.418 & 0.217 \\
\hline
\end{tabular}

Table 3. Maxima of $F_{\mathrm{L}} / F_{2}$ for different fits to $F_{2}$.

model and for the IIM model with parameters corresponding to the fit with $x<0.005$. We see that ratios $F_{\mathrm{L}} / F_{2}$ are in fact almost model-independent. This is further confirmed in Table 3 where we collect the maximal value of $F_{\mathrm{L}} / F_{2}$ for the GBW and IIM parametrizations.

One should note that each curve in Fig. 8 corresponds to a different definition of scaling variable $\tau$, so one cannot superimpose experimental data on that plot. This is done in Fig. 9 where we plot $F_{\mathrm{L}} / F_{2}$ for the unrestricted fits of the GBW and IIM models corresponding to the first rows of Table 1 and 2 respectively. The errors of the ratio have been calculated neglecting correlation between errors of $F_{2}$ and $F_{\mathrm{L}}$ :

$$
\Delta\left(\frac{F_{\mathrm{L}}}{F_{2}}\right)=\frac{F_{\mathrm{L}}}{F_{2}} \sqrt{\left(\frac{\Delta F_{\mathrm{L}}}{F_{L}}\right)^{2}+\left(\frac{\Delta F_{2}}{F_{2}}\right)^{2}}
$$

This procedure overestimates the errors, however, given the fact that $F_{L}<$ $0.27 F_{2}$ and that experimentally absolute errors of $F_{\mathrm{L}}$ are $2-10$ times larger than $\Delta F_{2}$, the error of the ratio $(19)$ is determined to very high precision by $\Delta F_{\mathrm{L}}$ alone. We shall come back to this point later.
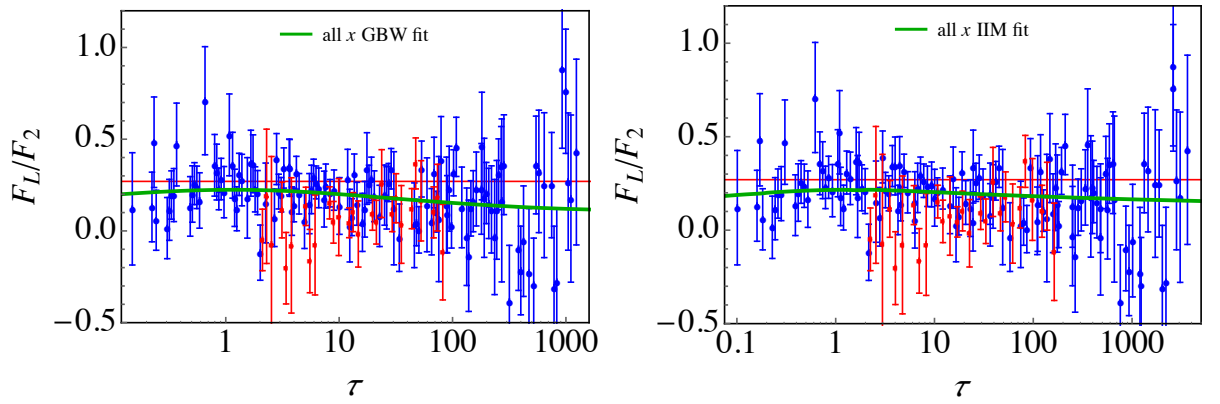

Fig. 9. Ratios $F_{\mathrm{L}} / F_{2}$ plotted as functions of scaling variable $\tau$. Straight line corresponds to the EMNS bound. Solid line in the left panel corresponds to the unrestricted GBW fit of Tab. 1 (first row), and in the right panel to the unrestricted IIM fit of Tab. 2 (first row). 
We can see from Figs. 8 and 9 that for realistic $\sigma_{\mathrm{dp}}$ theoretical predictions lie below the EMNS bound. Indeed, we see that the maximum of $F_{\mathrm{L}} / F_{2}$ is of the order $0.216-0.224$ and only slightly varies from fit to fit. This is illustrated in Table 3. A question arises whether data points - which for some values of $\tau$ exceed the EMNS bound - are indeed, as suggested by the authors of Ref. [8, saturating bound (11), being as a consequence incompatible with the dipole model. To this end we have simply calculated $\chi^{2}$ of $F_{\mathrm{L}} / F_{2}$ for the unrestricted GBW fit and obtained very good result: 0.7425 . To check whether this value is affected by the fact that we have not taken into account correlations between errors of $F_{\mathrm{L}}$ and $F_{2}$, we have recalculated $\chi^{2}$ neglecting $F_{2}$ errors, which gives $\chi^{2}$ that changes by less than $1 \%$. Therefore indeed, as already mentioned above, $\chi^{2}$ value is totally driven by the large errors of $F_{\mathrm{L}}$.

It is interesting to check whether an overall shift of the dipole model prediction for $F_{\mathrm{L}} / F_{2}$ would improve agreement with the data. In this way we shall have a quantitative measure of the quality of the dipole model prediction for the ratio $F_{\mathrm{L}} / F_{2}$ and also an indication how much room is there for the higher order corrections that we are going to discuss in Sect. 5 . To this end we use the GBW fits allowing for arbitrary normalization of the ratio

$$
\frac{F_{\mathrm{L}}}{F_{2}} \rightarrow \mathcal{N} \frac{F_{\mathrm{L}}}{F_{2}}
$$

and calculate $\chi^{2} /$ d.o.f. (assuming one degree of freedom, namely $\mathcal{N}$ ) as a function of $\mathcal{N}$. This is illustrated in Fig. 10 for two GBW fits of Table 1: unrestricted $x$ and $x<0.0005$. We see that depending on the fit the data prefers $\mathcal{N}$ sligthly smaller (unrestricted $x$ ) or sligthly larger $(x<0.0005)$ than 1 . These are negligible changes and therefore at this stage we conclude that the data for the ratio $F_{\mathrm{L}} / F_{2}$ is compatible with the dipole model. One should perhaps remind here again, that this ratio is quite stable, even for fits to $F_{2}$ that are visibly different.

One can also see that lowering $\mathcal{N}$ by $20 \%$, which would be requried by taking into account charm mass effects (c.f. discussion at the end of Sect. 2) increases $\chi^{2}$, but only by a small ammount. Of course the detialed study of charm mass effects would require to go beyond GS used in this analysis - which in turn would result not only in the change of $\mathcal{N}$, but also in the change of the shape of $F_{\mathrm{L}} / F_{2}$ - but no drastic difference to the present analysis should be expected.

\section{EMNS bound and higher Fock states}

In the dipole model the virtual photon dissociates into a $\bar{q} q$ pair which subsequently interacts with the proton target. However, it is clear that 

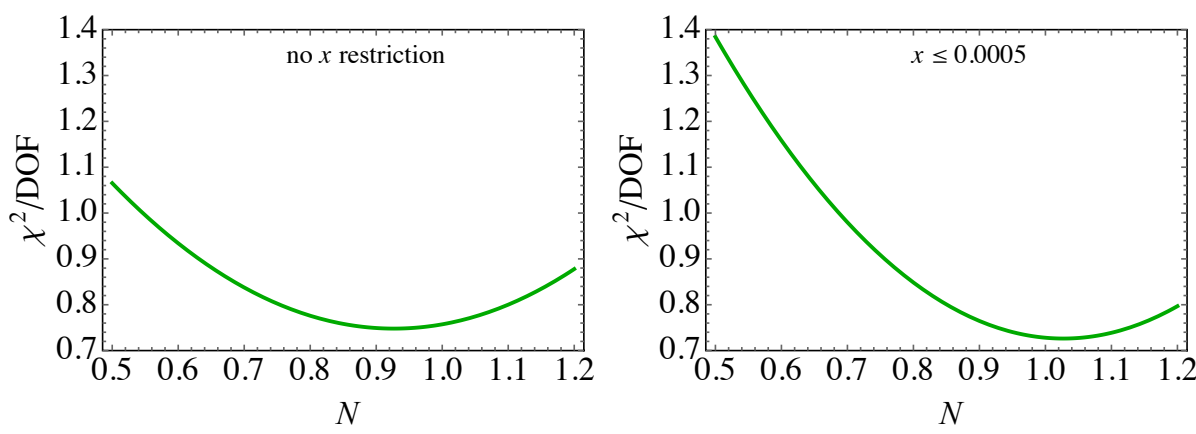

Fig. 10. Change of $\chi^{2}$ of $\mathcal{N} \times F_{\mathrm{L}} / F_{2}$ as a function of $\mathcal{N}$ for the GBW fits from Table 1 for unrestricted $x$ (left) and $x<0.0005$ (right).

higher Fock states have to contribute as well, similarly to the diffractive DIS where the next Fock component, namely the $\bar{q} q g$ state, is dominant at small $\beta$ [10]. Full calculations of the $\bar{q} q g$ component of the photon wave function have recently appeared in the literature [14, 15, 16, however they have not been yet applied to the phenomenological analysis of DIS.

Structure functions in the dipole model are given as an expansion

$$
F_{2, L}\left(x, Q^{2}\right)=F_{2, L}^{(\bar{q} q)}\left(x, Q^{2}\right)+F_{2, L}^{(\bar{q} q g)}\left(x, Q^{2}\right)+\ldots
$$

The EMNS bound (11) derived in Sect. 2 is in fact valid only for the first component of (21)

$$
G=\frac{F_{\mathrm{L}}^{(\bar{q} q)}\left(x, Q^{2}\right)}{F_{2}^{(\bar{q} q)}\left(x, Q^{2}\right)}<g_{\max }=0.27 .
$$

One should note, however, that at this order of perturbative expansion the loop corrections to the leading order Fock component may change the value of the bound (22). Similarly for the next Fock component we would have:

$$
H=\frac{F_{\mathrm{L}}^{(\bar{q} q g)}\left(x, Q^{2}\right)}{F_{2}^{(\bar{q} q g)}\left(x, Q^{2}\right)}<h_{\max }
$$

where

$$
0 \leq h_{\max } \leq 1 .
$$


Up to this order one can derive the modified EMNS bound:

$$
\begin{aligned}
\frac{F_{\mathrm{L}}\left(x, Q^{2}\right)}{F_{2}\left(x, Q^{2}\right)} & =\frac{F_{\mathrm{L}}^{(\bar{q} q)}\left(x, Q^{2}\right)+F_{\mathrm{L}}^{(\bar{q} q g)}\left(x, Q^{2}\right)}{F_{2}^{(\bar{q} q)}\left(x, Q^{2}\right)+F_{2}^{(\bar{q} q g)}\left(x, Q^{2}\right)} \\
& <\frac{g_{\max } F_{2}^{(\bar{q} q)}\left(x, Q^{2}\right)+h_{\max } F_{2}^{(\bar{q} q g)}\left(x, Q^{2}\right)}{F_{2}^{(\bar{q} q)}\left(x, Q^{2}\right)+F_{2}^{(\bar{q} q g)}\left(x, Q^{2}\right)} \\
& =g_{\max } \frac{1+\delta \varepsilon\left(x, Q^{2}\right)}{1+\varepsilon\left(x, Q^{2}\right)}
\end{aligned}
$$

where

$$
\delta=\frac{h_{\max }}{g_{\max }}, \quad 0 \leq \delta \leq \frac{1}{g_{\max }} \simeq 3.7
$$

and

$$
\varepsilon\left(x, Q^{2}\right)=\frac{F_{2}^{(\bar{q} q g)}\left(x, Q^{2}\right)}{F_{2}^{(\bar{q} q)}\left(x, Q^{2}\right)} .
$$

Since the dipole model with $\bar{q} q$ component only describes $F_{2}$ rather well over the wide kinematical range, we do not expect $\varepsilon$ to be large. For the purpose of the present analysis we assume that $\varepsilon$ does not exceed $20 \%$.

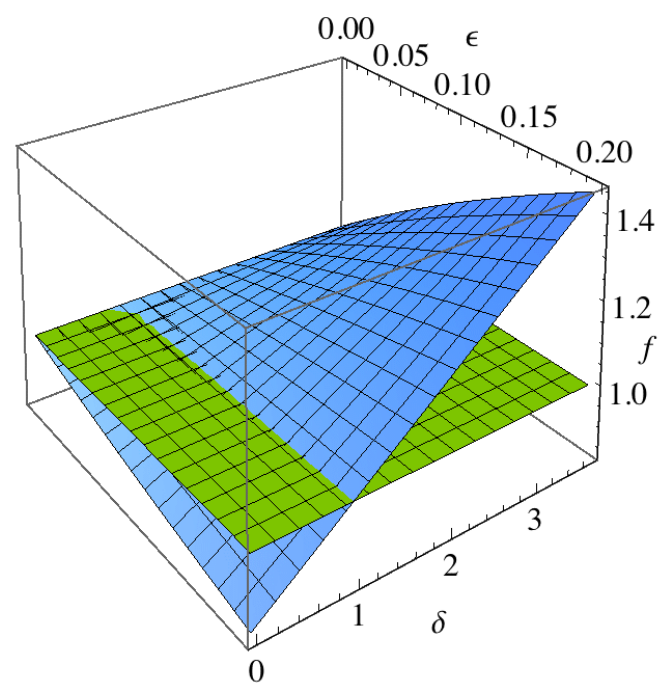

Fig. 11. Modification factor $f$ of Eq. 227) as a function of $\epsilon$ and $\delta$. Light green plane corresponds to $f=1$.

In order to change the value of the EMNS bound we need the modification factor

$$
f=\frac{1+\varepsilon \delta}{1+\varepsilon}
$$


to be significantly different from 1 . In Fig. 11 we plot $f(\varepsilon, \delta)$ for $0 \leq \varepsilon \leq 0.2$ and $0 \leq \delta \leq 1 / g_{\max }$. We see from Fig. 11 and from Eq. (27) that $f=1$ for $\delta=1$ and that $f$ gets smaller than 1 if $\delta<1$ and $f>1$ for $\delta>1$, and that the difference $|f-1|$ is growing with $\varepsilon$. From Fig. 11 one may conclude that the modification of the EMNS bound by more than $\pm 20 \%$ might be rather difficult. Most probably higher Fock components would modify (11) by less than $10 \%$, but to quantify this statement one needs to calculate explicitly $h_{\max }$ which is beyond the scope of the present paper. One should note at this point that in this case an inclusion of mass corrections due to the charm and possibly bottom quarks should be included, as these corrections would be of the same order or even larger than a contribution from the higher Fock components.

To conclude this Section let us only remark that $\varepsilon$ depends on kinematical variables, so one cannot exclude a'priori a situation that there exists a kinematical corner where a correction to (11) is of importance.

\section{Conclusions}

Dipole model offers effective and intuitive description of deep inelastic scattering which goes beyond leading twist approximation. In this paper we have used different variants of the dipole model, i.e. two different forms of photon-proton cross-section fitted to $F_{2}$ data over different ranges of Bjorken $x$ 's. We have decided to perform our new fits in the kinematical range where both $F_{2}$ and $F_{\mathrm{L}}$ have been measured. This kinematical region does not extend to very small $x$ 's as it is in the case of the recently published combined HERA data [19] and therefore fitted parameters are different than the ones of global fits. For the same reason the IIM model [18] that has been specifically devised for low $x$ region gives larger $\chi^{2}$ than the simplest version of the the GBW model [9], which quite satisfactorily describes $F_{2}$.

In order to fit model parameters we have used the property of geometrical scaling, which boils down to the fact that data points of the same $Q^{2}$ but different $x$ 's disperse when plotted in terms of scaling variable $\tau$ and fall on one line (compare Figs. 1 and e.g. 33. We have found that GS is present both in the case of $F_{2}$ and $F_{\mathrm{L}}$ as well. One should, however, take this property with care for the present set of data, since for each value of $Q^{2}$ only a few points of different Bjorken $x$ 's have been measured. This $x-Q^{2}$ correlation of the DIS data, particularly pronounced in the present case of $F_{\mathrm{L}}$, is a common obstacle in deriving firm conclusions on the quality of GS. In this context it is also worth mentioning that there exist different sources of GS. The first one is related to the genuine property of the initial state of the proton target described theoretically by the Color Glass Condensate formalism [20] and nonlinearities in the parton evolution [21], which are of 
importance at small values of Bjorken $x$. The other one is a property of the linear parton evolution equations [22], which build GS at larger $Q^{2}$ and at not necessarily very small $x$ 's.

We have next compared dipole model predictions - with parameters fixed by fits to $F_{2}$ - with $F_{\mathrm{L}}$ data. Here, due to large experimental errors, agreement is quite good. Next we have studied ratio of the structure functions $G=F_{\mathrm{L}} / F_{2}$ for which in the dipole model there exists a strict bound $g_{\max }=0.27$ (1) derived in Ref. [8. We have rederived (1) with the help of geometrical scaling. We have also shown that in the dipole models discussed above $g_{\max }^{\mathrm{dp}} \approx 0.216-0.224$ which is approximately $18.5 \%$ below the EMNS bound. Different fits give very similar ratio $g$, which is only residually dependent on the values of fit parameters (this concerns only the IIM dipole model, since ratio $F_{\mathrm{L}} / F_{2}$ is parameter independent in the GBW case). Comparing $G=F_{\mathrm{L}} / F_{2}$ with the data we have established that the GBW model reproduces $G$ with high precision. We do not see any tension between the data and the dipole model as far as ratio $G$ is concerned, even if charm mass effects, which lower the EMNS bound, are taken into account.

Dipole model and the EMNS bound discussed so far rely on the first Fock component of the photon wave function. Including higher Fock components, like a $\bar{q} q g$ state, might in principle change theoretical prediction for $g_{\max }$. For this to happen, longitudinal part of the $\bar{q} q g$ state compared to the transverse one has to be significantly different than in the $\bar{q} q$ case.

\section{Acknowledgments}

The authors want to thank Aharon Levy for bringing to their attention new data on $F_{\mathrm{L}}$ and for an access to this data prior to publication. M.P. thanks Otto Nachtmann for a conversation that started this project and for remarks on the final version of the manuscript. We thank Guillaume Beuf for bringing to our attention Refs. [15, 16]. The research of M.P. has been supported by the Polish NCN grants 2011/01/B/ST2/00492 and 2014/13/B/ST2/02486. 


\section{REFERENCES}

[1] V. Andreev et al. [H1 Collaboration], Eur. Phys. J. C 74 (2014) 2814 arXiv:1312.4821 [hep-ex]].

[2] H. Abramowicz et al. [ZEUS Collaboration], arXiv:1404.6376 [hep-ex].

[3] F. D. Aaron et al. [H1 Collaboration], Phys. Lett. B 665 (2008) 139 arXiv:0805.2809 [hep-ex]] and Eur. Phys. J. C 71 (2011) 1579 arXiv:1012.4355 [hep-ex]].

[4] A. Levy, talk at QCD Moriond 2014, arXiv:1405.3753 [hep-ex].

[5] C. G. Callan, Jr. and D. J. Gross, Phys. Rev. Lett. 22 (1969) 156.

[6] C. Ewerz and O. Nachtmann, Phys. Lett. B 648 (2007) 279 hep-ph/0611076.

[7] C. Ewerz, A. von Manteuffel and O. Nachtmann, Phys. Rev. D 77 (2008) 074022 arXiv:0708.3455 [hep-ph]].

[8] C. Ewerz, A. von Manteuffel, O. Nachtmann and A. Schöning, Phys. Lett. B 720 (2013) 181 arXiv:1201.6296 [hep-ph]].

[9] K.J. Golec-Biernat and M. Wüsthoff, Phys. Rev. D 59 (1998) 014017 hepph/9807513.

[10] K.J. Golec-Biernat and M. Wüsthoff, Phys. Rev. D 60 (1999) 114023 hepph/9903358.

[11] C. Ewerz and O. Nachtmann, Annals Phys. 322 (2007) 1670 hep-ph/0604087.

[12] A.M. Stasto, K.J. Golec-Biernat, J. Kwiecinski, Phys. Rev. Lett. 86 (2001) 596.

[13] M. Praszalowicz and T. Stebel, JHEP 1303 (2013) 090 arXiv:1211.5305 [hep$\mathrm{ph}]$.

[14] I. Balitsky and G. A. Chirilli, Phys. Rev. D 83 (2011) 031502 arXiv:1009.4729 [hep-ph]] and Phys. Rev. D 87 (2013) 1, 014013 [arXiv:1207.3844 [hep-ph]].

[15] G. Beuf, Phys. Rev. D 85 (2012) 034039 arXiv:1112.4501 [hep-ph]].

[16] R. Boussarie, A. V. Grabovsky, L. Szymanowski and S. Wallon, JHEP 1409 (2014) 026 [arXiv:1405.7676 [hep-ph]].

[17] See e.g. Chapter 9 in V. Baronne and E. Predazzi, High-Energy Particle Diffraction, Springer 2002.

[18] E. Iancu, K. Itakura and S. Munier, Phys. Lett. B 590 (2004) 199 hep$\mathrm{ph} / 0310338$.

[19] F. D. Aaron et al. [H1 and ZEUS Collaboration], JHEP 1001 (2010) 109 arXiv:0911.0884 [hep-ex]].

[20] L. D. McLerran, and R. Venugopalan, Phys. Rev. D49, 2233 (1994), Phys. Rev. D49, 3352 (1994), and Phys. Rev. D50, 2225 (1994).

[21] S. Munier and R. B. Peschanski, Phys. Rev. Lett. 91 (2003) 232001 hepph/0309177], and Phys. Rev. D 69 (2004) 034008 [hep-ph/0310357].

[22] F. Caola and S. Forte, Phys. Rev. Lett. 101 (2008) 022001 arXiv:0802.1878 [hep-ph]]. 